ARTICLE

\title{
Directional interlayer spin-valley transfer in two-dimensional heterostructures
}

John R. Schaibley¹, Pasqual Rivera1, Hongyi Yu², Kyle L. Seyler ${ }^{1}$, Jiaqiang Yan ${ }^{3,4}$, David G. Mandrus ${ }^{3,4,5}$, Takashi Taniguchi $^{6}$, Kenji Watanabe ${ }^{6}$, Wang $\mathrm{Yao}^{2} \&$ Xiaodong $\mathrm{Xu}^{1,7}$

Van der Waals heterostructures formed by two different monolayer semiconductors have emerged as a promising platform for new optoelectronic and spin/valleytronic applications. In addition to its atomically thin nature, a two-dimensional semiconductor heterostructure is distinct from its three-dimensional counterparts due to the unique coupled spin-valley physics of its constituent monolayers. Here, we report the direct observation that an optically generated spin-valley polarization in one monolayer can be transferred between layers of a two-dimensional $\mathrm{MoSe}_{2}-\mathrm{WSe}_{2}$ heterostructure. Using non-degenerate optical circular dichroism spectroscopy, we show that charge transfer between two monolayers conserves spin-valley polarization and is only weakly dependent on the twist angle between layers. Our work points to a new spin-valley pumping scheme in nanoscale devices, provides a fundamental understanding of spin-valley transfer across the two-dimensional interface, and shows the potential use of two-dimensional semiconductors as a spin-valley generator in two-dimensional spin/valleytronic devices for storing and processing information.

\footnotetext{
${ }^{1}$ Department of Physics, University of Washington, Seattle, Washington 98195, USA. ${ }^{2}$ Department of Physics and Center of Theoretical and Computational Physics, University of Hong Kong, Hong Kong, China. ${ }^{3}$ Materials Science and Technology Division, Oak Ridge National Laboratory, Oak Ridge, Tennessee 37831, USA. ${ }^{4}$ Department of Materials Science and Engineering, University of Tennessee, Knoxville, Tennessee 37996, USA. ${ }^{5}$ Department of Physics and Astronomy, University of Tennessee, Knoxville, Tennessee 37996, USA. ${ }^{6}$ Advanced Materials Laboratory, National Institute for Materials Science, Tsukuba, Ibaraki 305- 0044, Japan. ${ }^{7}$ Department of Materials Science and Engineering, University of Washington, Seattle, Washington 98195, USA. Correspondence and requests for materials should be addressed to J.R.S. (email: johnschaibley@email.arizona.edu) or to X.X. (email: xuxd@uw.edu)
} 
S pin initialization is a crucial operation for spintronic devices which require a net spin polarization for reading, writing and transferring information ${ }^{1}$. Two-dimensional semiconductors, such as monolayer $\mathrm{MoSe}_{2}$ and $\mathrm{WSe}_{2}$, have recently emerged as a new spin/valleytronic platform ${ }^{2-5}$. Their inversion-asymmetric honeycomb lattice structures give rise to two energy degenerate but inequivalent $(+\mathrm{K}$ and $-\mathrm{K})$ momentum-space valleys, forming a pseudospin system analogous to real electron $\operatorname{spin}^{6}$. Due to strong spin-orbit coupling, the valley pseudospin is locked to the real spin orientation $^{6}$. Since flipping an electron spin requires a simultaneous flip of a valley pseudospin, free carrier spin-valley polarization at the band edge is expected to be robust and long lived, which has recently been measured to be on order of 1-100 ns (refs 7,8). Large spin-valley polarizations associated with excitons have been generated by circularly polarized light excitation through a valley dependent optical selection rule $3,6,9-11$. However, excitonic spin-valley polarization in a monolayer does not last long compared with free carriers due to the picosecond timescale of the valley exciton depolarization time, which arises from the electron-hole exchange interaction $^{3,12-14}$ and the ultrafast decay time of the exciton itself $^{15,16}$. In addition, it is not clear how to exploit a monolayer system as a spin generator to supply optically generated spin-valley polarization to a different physical system.

2D semiconductor heterostructures formed by stacking two monolayers on top of each other can be designed to realize new spin-valley systems with important advantages over individual monolayers. It has been established that $\mathrm{WX}_{2}-\mathrm{MoX}_{2}$ (where $\mathrm{X}=\mathrm{S}, \mathrm{Se}$ ) heterostructures have a type-II band alignment ${ }^{17,18}$, which leads to ultrafast charge transfer between layers and tunable photodetectors ${ }^{19,20}$. Such spatial separation of electrons and holes suppresses ultrafast electron-hole recombination ${ }^{21-24}$ and their exchange interaction ${ }^{21-26}$, both of which limit the practical application of optical spin-valley orientation in monolayers $3,27,28$. Very recently, helicity-dependent photoluminescence (PL) measurements of interlayer excitons revealed spin-valley polarization lifetimes exceeding tens of nanoseconds ${ }^{26}$, showing that the spatial separation of electrons and holes indeed provides a powerful approach towards practical spin-valleytronics. However, interlayer exciton effects were accompanied by complicated electron-hole relaxation pathways and the effect of the twist angle between the two layers ${ }^{25}$, which complicate the quantitative analysis of spin-valley polarization from the polarization resolved interlayer exciton PL. In addition, interlayer exciton PL studies were limited to small twist angle samples only, because the electron-hole momentum mismatch in large twist angle heterostructures strongly suppresses interlayer exciton light emission. All of these limitations obscured a clear understanding of the unique spin-valley properties of $2 \mathrm{D}$ semiconductor heterostructures, especially the transport of spin-valley polarized free carriers across the $2 \mathrm{D}$ layer interface.

In this work, by applying polarization resolved non-degenerate nonlinear optical spectroscopy, we provide a direct probe of interlayer spin-valley polarization transfer in a model $2 \mathrm{D}$ heterostructure with varying twist angles formed by monolayer $\mathrm{MoSe}_{2}$ and $\mathrm{WSe}_{2}$. By optically exciting an intralayer exciton spin-valley polarization in one layer and probing the intralayer neutral and charge excitons in different layers, we demonstrate that the subsequent interlayer charge transfer is directional and conserves spin, that is, spin polarization transfer leads to polarized hole spins in $\mathrm{WSe}_{2}$ and electron spins in $\mathrm{MoSe}_{2}$ (Fig. 1a). We find that the spin-valley polarization transfer has only a weak dependence on twist angles in the heterobilayer. Our results realize directional pumping of spin-valley polarized carrier spins into individual layers of a $2 \mathrm{D}$ heterostructure by harnessing the coupled spin-valley physics of the constituent monolayers ${ }^{6}$.

\section{Results}

Sample fabrication and electronic structure. The $\mathrm{MoSe}_{2}-\mathrm{WSe}_{2}$ heterostructures were fabricated from independently isolated, exfoliated monolayers (see Fig. 1b). To investigate the effect of heterostructure twist angle, we first measured the crystal axes of individual monolayers by polarization resolved and phase-sensitive second-harmonic generation spectroscopy ${ }^{29-32}$ (see Supplementary Fig. 1 and Supplementary Note 1). The monolayers were then assembled into heterostructures using a dry transfer stamping technique ${ }^{33}$ with known twist angle. Results from heterostructures with non-zero twist angels are presented in Supplementary Note 2 and Supplementary Figs 2 and 3. The sample in the main text has a twist angle near $0^{\circ}$, where the valleys from the different layers are nearly aligned in momentum space (Fig. 1c). The lowest conduction band is located in the $\mathrm{MoSe}_{2}$ and the highest valence band in $\mathrm{WSe}_{2}$. Within each monolayer, $\sigma \pm$ circularly polarized light couples to transitions in the $\pm K$ valley only. The high quality of our heterostructure was confirmed by observing a strong $\mathrm{PL}$ quenching of the intralayer excitons, and the observation of interlayer excitons (see Supplementary Fig. 4), where Coulombbound electrons and holes are localized in opposite layers ${ }^{24}$.

Nonlinear excitonic response of the heterostructure. We first determined the energy position of intralayer excitons by performing energy resolved continuous-wave differential transmission (DT) or differential reflection (DR) spectroscopy ${ }^{34}$ This is a two beam pump-probe technique which measures the difference of the probe transmission or reflection when the pump is on and off. The experiments in the main text were all performed on the same heterostructure mounted on sapphire. Additional measurements were performed on different heterostructures on $\mathrm{SiO}_{2}$ substrates and are in qualitative agreement with the data presented in the main text (see Supplementary Note 2). The experiments were performed at $30 \mathrm{~K}$, unless otherwise specified.

The degenerate DT spectrum of a heterostructure is shown in Fig. 1d with cross-circularly polarized pump and probe. Compared with the DT spectrum from individual monolayers, we see the intralayer exciton resonances in the heterostructure are consistent with the spectral positions of isolated monolayers with a $\sim 20 \mathrm{meV}$ redshift and broader linewidth. We attribute the $\sim 20 \mathrm{meV}$ redshift to a reduction in the intralayer exciton bandgaps due to the coupling between layers. The linewidth broadening is attributed to the charge transfer between the layers, which leads to an extra relaxation channel for the intralayer excitons $^{35}$. The resonance line shapes of the degenerate DT spectrum consist of a pump-induced increase to the probe transmission at high energy and a pump-induced absorption at low energy. Note that the low-energy pump-induced absorption feature is stronger for the $\mathrm{MoSe}_{2}$ layer compared with $\mathrm{WSe}_{2}$. We attribute this difference to the different oscillator strengths of different charged exciton species in each layer (see Supplementary Note 3).

Demonstration of interlayer charge transfer. To establish interlayer carrier transfer, we performed two-colour nondegenerate DR and DT measurements. Both types of measurements were performed on the same sample and the data are qualitatively similar. We use the DT data exclusively in curve fitting to avoid the interference effects that arise from the substrate reflection in the DR measurements. Figure 2 a shows the 


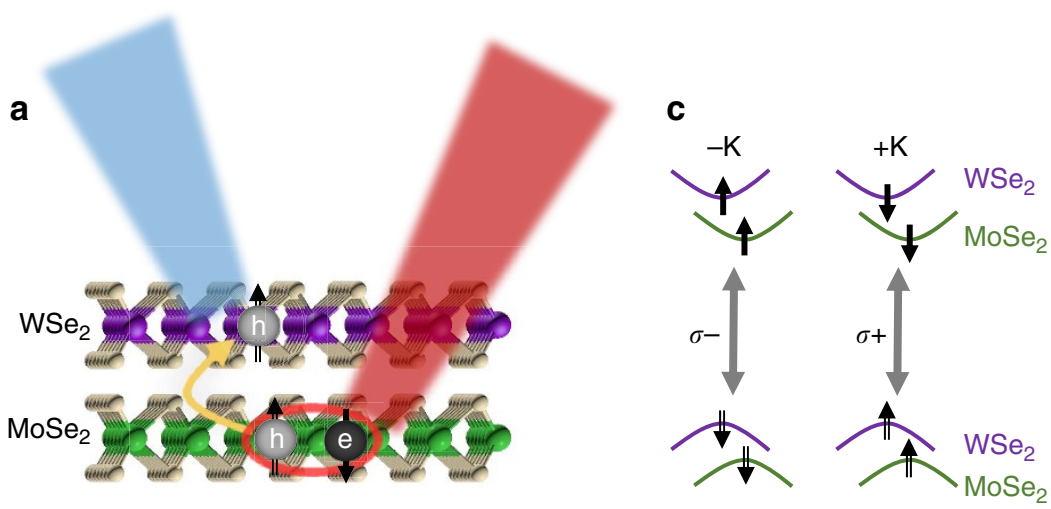

b

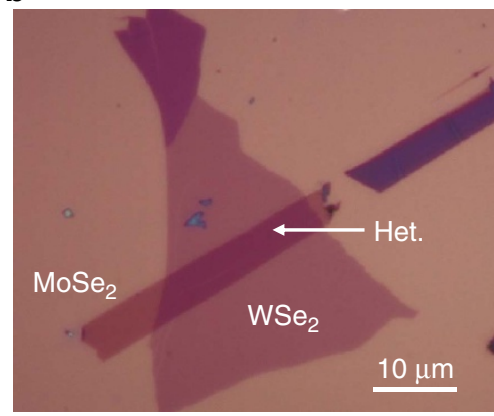

d

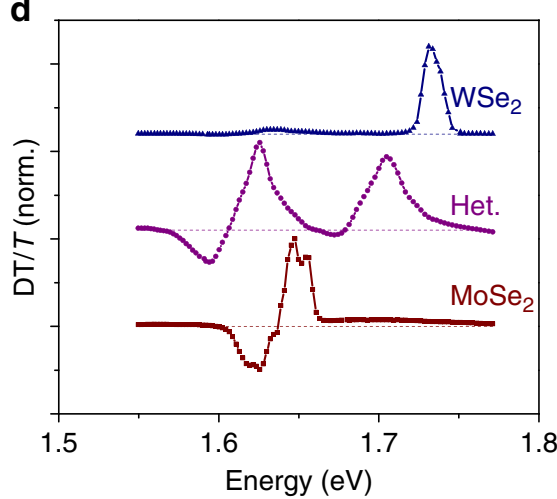

Figure 1 | Interlayer spin-valley physics. (a) Depiction of the experiment. Spin-valley polarized excitons are resonantly injected in the MoSe 2 layer with a polarized laser (red). The hole transfers to the WSe $e_{2}$ layer where its spin-valley polarization is measured with another polarized laser (blue), resonant with the $\mathrm{WSe}_{2}$ excitons. The black arrows depict the real spin of the electrons and holes. (b) Optical microscope image of a MoSe ${ }_{2}-\mathrm{WSe}_{2}$ heterostructure (Het.) on $\mathrm{SiO}_{2}$, showing the different sample regions. (c) The 8-band model of the $+\mathrm{K}$ and $-\mathrm{K}$ valleys for a nearly aligned MoSe $\mathrm{C}_{2}-\mathrm{WSe} \mathrm{h}_{2}$ heterostructure, showing the valley dependent optical selection rules ( $\sigma \pm$ for $\pm \mathrm{K}$ valley) and real spins (black arrows) for electrons and holes. (d) Degenerate DT spectra from different sample regions for a $\mathrm{MoSe}_{2}-\mathrm{WSe}_{2}$ heterostructure on sapphire, which are normalized and stacked for comparison. The dashed lines correspond to $\mathrm{DT} / T=0$ for each spectrum. Due to the small isolated WSe $\mathrm{W}_{2}$ area used in the DT study, the laser beam could not completely avoid the heterobilayer region,

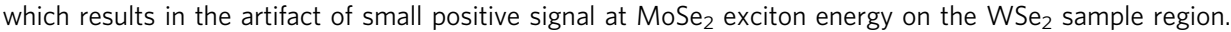

DR spectrum with co-circularly polarized pump and probe, where the pump is resonant with the lower energy $\mathrm{MoSe}_{2}$ exciton at $1.621 \mathrm{eV}$ while the probe laser scans over the $\mathrm{WSe}_{2}$ exciton resonance near $1.68 \mathrm{eV}$. The green curve shows an enhanced DR response from the heterostructure region. In comparison, the black curve shows the DR response when both pump and probe are focused on an isolated monolayer $\mathrm{WSe}_{2}$ region which shows a negligible DR response when the pump energy is fixed at the $\mathrm{MoSe}_{2}$ exciton resonance. In the heterostructure, since the $\mathrm{MoSe}_{2}$ exciton has lower energy than $\mathrm{WSe}_{2}$, the observed DR response near the $\mathrm{WSe}_{2}$ exciton when pumping the $\mathrm{MoSe}_{2}$ exciton resonance is unlikely from the energy transfer from $\mathrm{MoSe}_{2}$ exciton. Rather, it is a result of charge transfer from $\mathrm{MoSe}_{2}$ to $\mathrm{WSe}_{2}$. Specifically, the hole is transferred from the $\mathrm{MoSe}_{2}$ valence band to the $\mathrm{WSe}_{2}$ valence band due to the type-II band alignment.

Demonstration of interlayer spin-valley polarization transfer. Interlayer spin-valley transfer was then investigated by performing polarization resolved DT experiments which measure the pump-induced circular dichroism (CD). The pump laser polarization and energy were chosen to only excite valley polarized excitons in the $\mathrm{MoSe}_{2}$ layer. The DT spectrum was measured for both co- (burgundy curve) and cross- (green curve) circularly polarized configurations for the probe scanning through the $\mathrm{WSe}_{2}$ excitons (Fig. $2 \mathrm{~b}$ ). The $\mathrm{CD}$ can be defined as the difference between the cross- and co-polarized DT spectra for either fixed pump or fixed probe polarization. Both yield similar results. For the convenience of our experimental configuration, we choose to fix the probe helicity while switching the pump helicity, that is, $\mathrm{CD}=\frac{\mathrm{DT}}{T}\left(\sigma_{\text {pump }}^{-}\right)-\frac{\mathrm{DT}}{T}\left(\sigma_{\text {pump }}^{+}\right)$, where the subscript denotes the pump beam, and $T$ is the probe transmission. As shown in Fig. 2c, the sign of the pump-induced CD response reverses for opposite probe helicities. The observed $\mathrm{CD}$ demonstrates a valley population imbalance, that is, the creation of spin-valley polarization in $\mathrm{WSe}_{2}$. We attribute this population imbalance to the pumping of polarized hole spins as depicted in Fig. 1a. Circularly polarized excitation resonantly pumps spin-valley polarized excitons in the $\mathrm{MoSe}_{2}$ layer, about $60 \mathrm{meV}$ below the $\mathrm{WSe}_{2}$ exciton energy. The spin polarized hole then transfers to the $\mathrm{WSe}_{2}+\mathrm{K}$ valence band, which gives rise to hole spin-valley polarization in $\mathrm{WSe}_{2}$ and electron spin-valley polarization in $\mathrm{MoSe}_{2}$. The observation of the CD response supports this picture.

We also demonstrate electron spin transfer from the $\mathrm{WSe}_{2}$ to the $\mathrm{MoSe}_{2}$ layer by resonantly pumping a WSe $\mathrm{W}_{2}$ spin-valley polarization and probing the $\mathrm{MoSe}_{2}$ excitons (Fig. 3). Similar phenomena, including the pump-induced CD is observed, whose sign depends on probe helicity. Since the $\mathrm{WSe}_{2}$ exciton has higher energy than $\mathrm{MoSe}_{2}$, the observed CD will have two contributions. One is due to the electron spin transfer from the $\mathrm{WSe}_{2}$ to the $\mathrm{MoSe}_{2}$ conduction band. The other is from the above resonance optical excitation of valley-polarized excitons directly in the $\mathrm{MoSe}_{2}$. To distinguish these two effects, we measured the DR response on the heterostructure when pumping at the $\mathrm{WSe}_{2}$ 

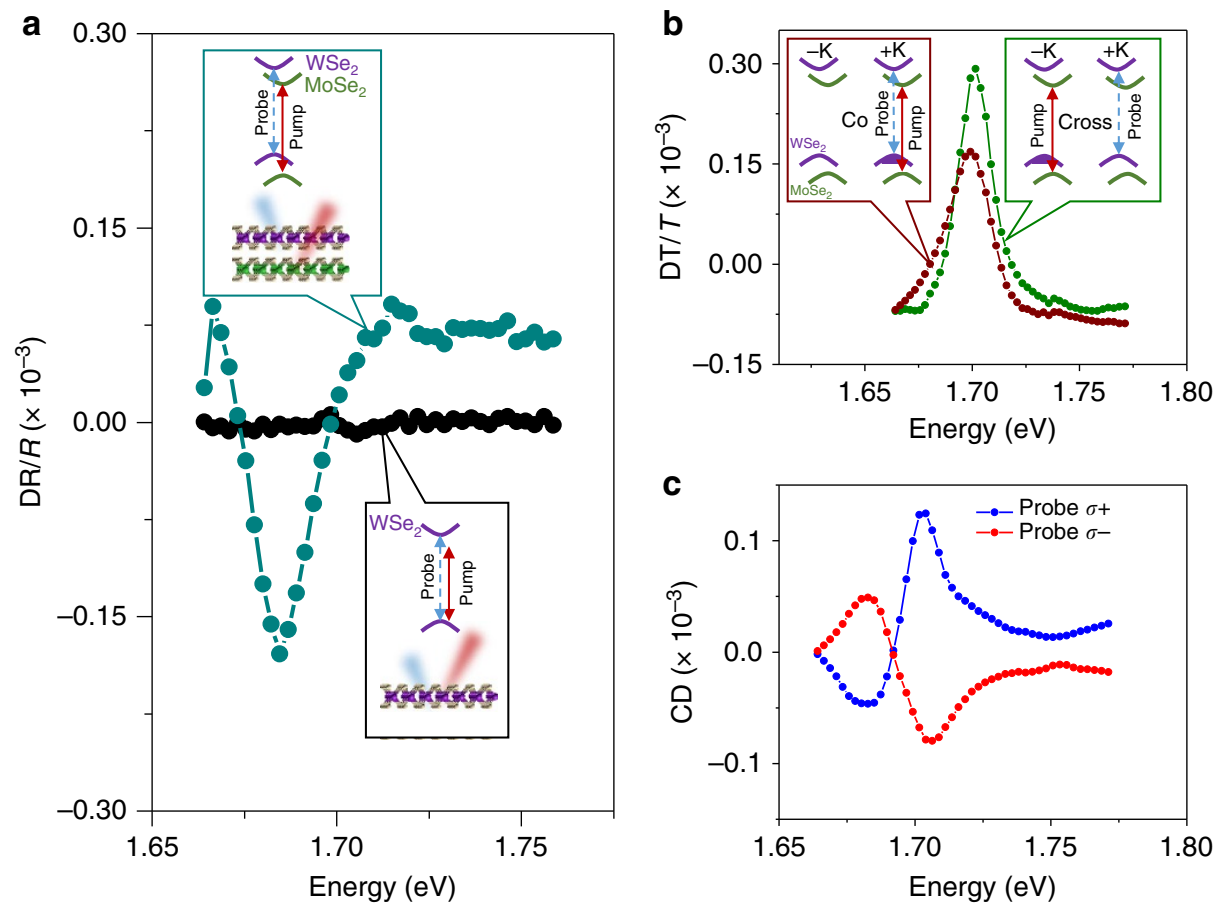

Figure 2 | Interlayer hole spin-valley polarization transfer. (a) Non-degenerate DR of a $\mathrm{MoSe}_{2}-\mathrm{WSe}_{2}$ heterostructure, and an isolated WSe $\mathrm{W}_{2}$ region on the sample. When pumping on the lower energy $\mathrm{MoSe}_{2}$ exciton resonance $(1.621 \mathrm{eV})$, there is a strong DR response corresponding to the WSe exciton (dark cyan), whereas the isolated $\mathrm{WSe}_{2}$ monolayer shows negligible DR response (black). Co-circularly polarized pump and probe is shown. The insets depict the pump-probe scheme. The pump is shown as a solid red line, and the probe is the dashed blue line. DR data were measured at $50 \mathrm{~K}$. (b) Co- (burgundy) and cross- (green) circularly polarized DT spectra of the $\mathrm{WSe}_{2}$ exciton resonances, when pumping the low-energy MoSe $\mathrm{S}_{2}$ exciton resonance at $1.621 \mathrm{eV}$. The insets show the pump and probe scheme, where the band filling of the WSe $\mathrm{W}_{2}$ valence is shown. The line shapes are discussed in the text. (c) Pump-induced $\mathrm{CD}$ of the $\mathrm{WSe}_{2}$ exciton resonances when pumping $\mathrm{MoSe}_{2}$ at $1.621 \mathrm{eV}$. CD highlights the differences between co- and cross-polarized DT responses. As expected, the sign of the CD response flips with probe (or pump) helicity.

resonance, and then repeated the measurement on the isolated monolayer $\mathrm{MoSe}_{2}$ region of the same sample (Supplementary Fig. 5). We observe a threefold enhancement of the DR response on the heterostructure region, which shows that electron transfer from the $\mathrm{WSe}_{2}$ to the $\mathrm{MoSe}_{2}$ dominates the DR response. We note that since the electron and hole spin are separated in opposite layers, the exchange interaction between the electron and hole spins is strongly suppressed. This will give rise to a long polarization lifetime $\mathrm{e}^{26}$ and contributes to the enhanced DR response.

Origin of the DT line shapes. We now turn to the discussion of the line shapes in the non-degenerate DT measurements (Figs $2 \mathrm{~b}$ and $3 \mathrm{a}$ ), which further support the picture of directional spin transfer. For simplicity, we focus on the explanation of data in Fig. 3a. Figure $3 \mathrm{c}-\mathrm{f}$ illustrate the origins of the line shapes by pumping at the $\mathrm{WSe}_{2}$ exciton resonance while probing the $\mathrm{MoSe}_{2}$ excitons. The DT spectra can be understood by taking the difference between the probe transmission spectrum with the pump on and off (solid orange and dashed blue curve of Fig. 3c,e). The co-polarized pump and probe (burgundy data) laser configuration is shown in the left inset of Fig. 3a. The inset depicts the pump (solid blue line) injecting $+\mathrm{K}$ polarized carriers in the $\mathrm{WSe}_{2}$ layer and the consequent electron transfer to the $+\mathrm{K}$ conduction band valley in the $\mathrm{MoSe}_{2}$ monolayer. The probe (dashed red line) measures the changes in transmission spectrum of the $+\mathrm{K} \mathrm{MoSe}_{2}$ excitons. Figure 3c,d depict the effects that dominate the co-polarized DT response. Because the conduction band is partially filled, phase-space filling leads to a blue shift of the transmission resonance, and the neutral exciton $\left(\mathrm{X}^{\circ}\right)$ oscillator strength is reduced (Fig. 3c). The inset to Fig. 3c depicts the DT signal calculated by taking the difference between the orange and dashed blue curves.

The cross-polarized pump and probe configuration (green data) is depicted in the right inset of Fig. 3a. Here, the pump (solid blue line) injects carriers into the $-\mathrm{K}$ valley of the $\mathrm{WSe}_{2}$ layer, and the subsequent electron transfer to the $-\mathrm{K}$ valley of the $\mathrm{MoSe}_{2}$. With this $-\mathrm{K}$ valley electron population, when the probe beam (dashed red line) excites electron-hole pairs in $+\mathrm{K}$ valley, negatively charged excitons $\left(\mathrm{X}^{-}\right)$can form (Fig. 3e,f). The crosspolarized DT spectrum can be understood by examining Fig. 3e, which shows the pump-induced changes to the cross-polarized probe transmission spectrum. Relative to the pump off case, a population of electrons in the $-\mathrm{K}$ valley decreases the $+\mathrm{K}$ cross-polarized probe transmission at $\mathrm{X}^{-}$resonance due to the increases of $\mathrm{X}^{-}$oscillator strength, and increases the transmission at the $\mathrm{X}^{\circ}$ resonance due to the decrease of $\mathrm{X}^{\circ}$ oscillator strength. The inset to Fig. 3e shows the corresponding crosspolarized DT spectrum. We note that the $30 \mathrm{meV}$ energy separation between the peak and dip in both the cross-polarized DT spectrum (green curve of Fig. 3a) and the CD spectra (Fig. 3b) is consistent with the binding energy of $\mathrm{X}^{-}$, and therefore further supports the picture of directional electron spin-valley transfer from $\mathrm{WSe}_{2}$ to $\mathrm{MoSe}_{2}$.

\section{Discussion}

We estimate the resulting spin-valley polarization of electrons in the $\mathrm{MoSe}_{2}$ layer by pumping the $\mathrm{WSe}_{2}$ resonance and comparing the relative co- and cross-circularly polarized DT responses of the $\mathrm{X}^{-}$in the $\mathrm{MoSe}_{2}$ layer. It has been demonstrated both experimentally ${ }^{4}$ and theoretically ${ }^{36}$ that the $\mathrm{X}^{-}$in $\mathrm{MoSe}_{2}$ is dominantly an intervalley charged exciton with the extra electron 

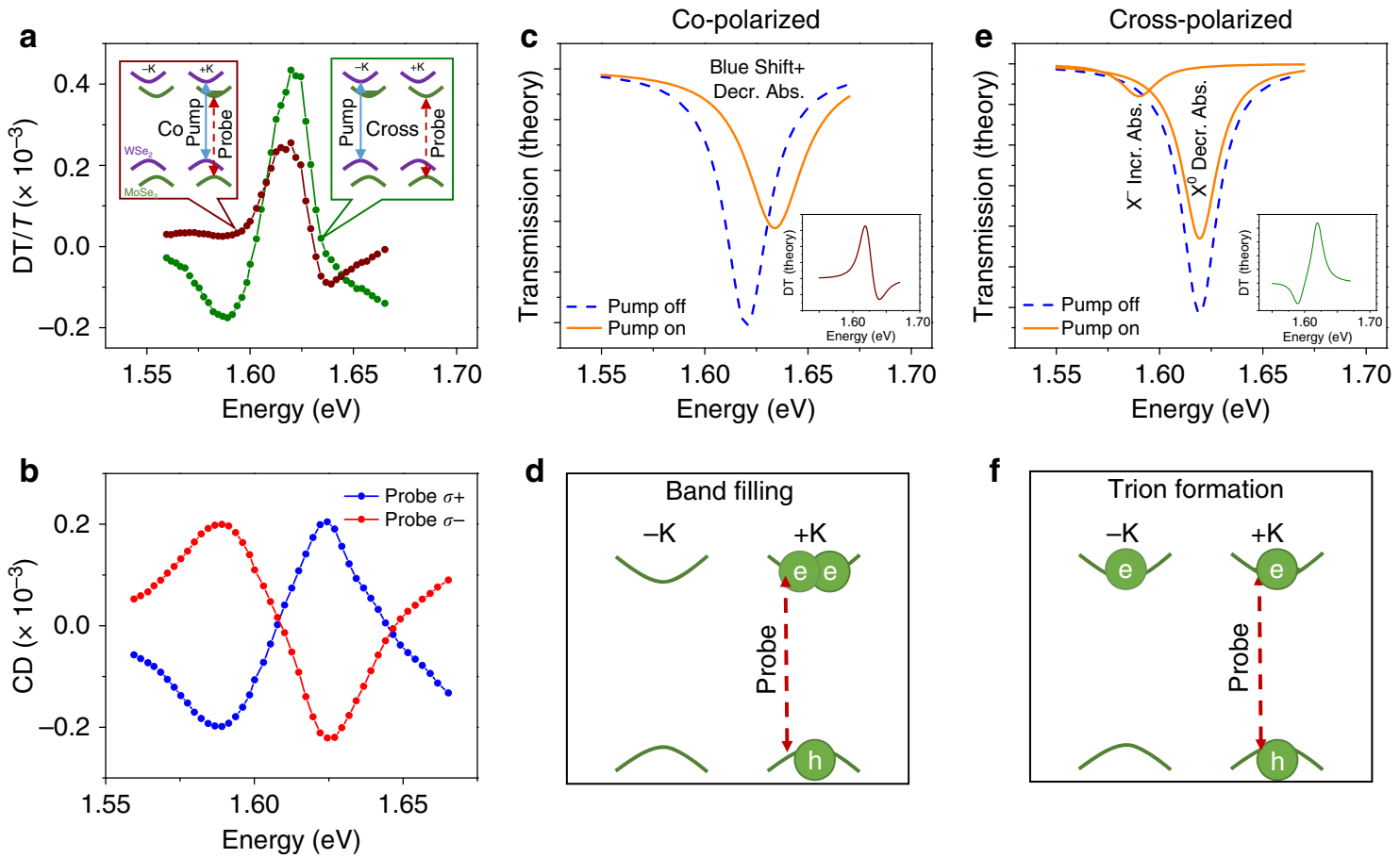

Figure 3 | Interlayer electron spin-valley polarization transfer. (a) Co- (burgundy) and cross- (green) circularly polarized DT spectra of the MoSe 2 exciton resonances, when pumping the higher energy $\mathrm{WSe}_{2}$ exciton resonance at $1.710 \mathrm{eV}$. The insets show the pump and probe scheme, where the pump is the solid blue line, and the probe is the dashed red line. Following the interlayer transfer of photo-excited electrons from WSe $\mathrm{W}_{2}$ to $\mathrm{MoSe}_{2}$, spin-valley polarized electrons are pumped into the $\mathrm{MoSe}_{2}$ layer. The band filling of the $\mathrm{MoSe}_{2}$ conduction band is shown. (b) The pump-induced $\mathrm{CD}$ of the $\mathrm{MoSe}_{2}$ exciton resonances when pumping $\mathrm{WSe}_{2}$ at $1.710 \mathrm{eV}$ flips sign with probe (or pump) helicity. (c-f) Theoretical explanations of the DT line shapes. (c,d) For co-polarized pump and probe, the polarized electrons populate the same valley that the probe measures. The dominant effect is a band filling effect, so that when the pump is on (orange curve in $\mathbf{c}$ ), the resonance is blue shifted and the exciton absorption is partially saturated, relative to the pump off case (blue dashed curve in c). In this co-polarized configuration, a charged exciton cannot form due to Pauli blocking. (e,f) For cross-polarized pump and probe, the polarized electrons populate the opposite valley that the probe measures. The dominant effect is charged exciton $\left(X^{-}\right)$formation, so that when the pump is on (orange curve in $\mathbf{e}$ ), the transmission is decreased at the $X^{-}$resonance, and increased at the neutral exciton $\left(X^{\circ}\right)$ resonance, relative to the pump off case (blue dashed curve in $\mathbf{e}$ ). The insets of $\mathbf{c}, \mathbf{e}$ show the difference between the modelled pump on (orange) and pump off (blue dashed) curves, corresponding to the theoretical DT spectra.

located in the lower conduction band of the opposite valley (see Supplementary Note 4 and Supplementary Fig. 6). The $\mathrm{X}^{-}$ formation and the magnitude of its corresponding DT signal measures the population of polarized electrons in the valley opposite the one being probed. Therefore, spin-valley polarization $(\rho)$ in the $\mathrm{MoSe}_{2}$ layer resulting from interlayer spin-valley transfer can be estimated by $\rho=\frac{\mathrm{DT} / T(\mathrm{cross})-\mathrm{DT} / T(\mathrm{co})}{\mathrm{DT} / T(\mathrm{cross})+\mathrm{DT} / T(\mathrm{cos})}$. Since the negative $\mathrm{MoSe}_{2}$ charged exciton resonance is spectrally isolated, it is not significantly influenced by signals arising from the other resonances (Fig. 3a). We fit single Lorentzians to the $\mathrm{MoSe}_{2}$ DT response near $\mathrm{X}^{-}(1.589 \mathrm{eV})$ for both co- and cross-circularly polarized pump and probe, and find that the ratio of the dip areas for the co-polarized response is $37 \%$ of the cross-polarized response (See Supplementary Fig. 7). This gives a 46\% electron spin-valley polarization in the $\mathrm{MoSe}_{2}$ layer. This estimation is consistent with previous measurements of the interlayer exciton in helicity-dependent PL measurements, which was reported to be limited by depolarization of the monolayer exciton before interlayer transfer ${ }^{26}$.

When pumping the $\mathrm{MoSe}_{2}$ and probing the $\mathrm{WSe}_{2}$ excitons, the charged exciton feature is also clear in the $\mathrm{CD}$ response (Fig. 2c). Fitting the $\mathrm{CD}$ spectra with a difference of two Lorentzians, we find that the energy separation between the peak and dip is approximately $19 \mathrm{meV}$, consistent with the binding energy of positively charged excitons $\left(\mathrm{X}^{+}\right)$in $\mathrm{WSe}_{2}$ (refs 2,3). This observation supports the picture of directional polarized hole spin transfer from $\mathrm{MoSe}_{2}$ to $\mathrm{WSe}_{2}$. However, due to the overlap of spectral features near the $\mathrm{WSe}_{2}$ positively charged exciton peak, we cannot accurately compare the co- and cross-circular DT responses of $\mathrm{X}^{+}$to estimate a hole spin-valley polarization in the $\mathrm{WSe}_{2}$ layer.

We also performed measurements on additional samples with varying twist angles (Supplementary Fig. 2). There are fine spectral features distinct from near zero twist angle samples, which require a future systematic study. However, both the sign and signal amplitude of the CD spectra are consistent for all twist angles, which implies that spin-valley conserved interlayer charge transport is robust for different twist angles.

Our results demonstrate that spin-valley polarized carriers can be efficiently transferred between layers, providing a novel method for optically injecting long-lived and spin-valley polarized carriers in either layer of heterostructures with arbitrary twist angles. We expect this scheme could be especially useful in recent proposals that seek to use atomically thin-bilayer systems for spintronic or valleytronic applications $s^{25}$, or as a platform to investigate bosonic quasiparticle effects with spin structures ${ }^{37}$.

\section{Methods}

Sample fabrication. The heterostructures were assembled using a polycarbonate film dry transfer technique. Supplementary Note 1 contains the methods used to determine the crystal axes. The sample in the main text was encapsulated in 5-10 $\mathrm{nm}$ thick hexagonal boron nitride and mounted on a $c$-axis sapphire substrate to allow for optical transmission measurements. 
Nonlinear optical measurements. The data shown in the main text were measured in a cold-finger cryostat. Two continuous-wave tunable Ti:sapphire lasers $\left(\mathrm{M}^{2}\right.$ SolsTiS) provided the pump and probe beams, which were each amplitude modulated with acousto-optic modulators at frequencies near $700 \mathrm{kHz}$. Both beams were actively intensity stabilized. A probe of $20 \mu \mathrm{W}$ and a pump of $40 \mu \mathrm{W}$ average power were used for all spectra in the main text. Polarizers and broadband waveplates were used to set the polarization of pump and probe, which were focused onto the sample with a microscope objective to a beam spot of $\sim 1 \mu \mathrm{m}$. The transmitted light was collected by a $15 \mathrm{~mm}$ spherical lens that was mounted in the cold finger of the cryostat. In the DR measurements, the reflected probe was collected with the objective. The pump beam was rejected with a crosspolarized set-up, or with a short or long pass filter. The probe was detected with an amplified silicon photodiode. The DT or DR signal was then measured with a phase-sensitive lock-in amplifier which was locked to the difference between the pump and probe modulation frequencies. The transmitted $(T)$ or reflected $(R)$ probe power was measured simultaneously with the DT or DR signal while the pump was modulated and used to normalize the DT/T or DR/R response.

Data availability. The authors declare that all of the data supporting the findings of this study are available within the article and its Supplementary Information file.

\section{References}

1. Wolf, S. et al. Spintronics: a spin-based electronics vision for the future. Science 294, 1488-1495 (2001).

2. Ross, J. S. et al. Electrical control of neutral and charged excitons in a monolayer semiconductor. Nat. Commun. 4, 1474 (2013).

3. Jones, A. M. et al. Optical generation of excitonic valley coherence in monolayer $\mathrm{WSe}_{2}$. Nat. Nanotech. 8, 634-638 (2013).

4. Li, Y. et al. Valley splitting and polarization by the Zeeman effect in monolayer $\mathrm{MoSe}_{2}$. Phys. Rev. Lett. 113, 266804 (2014).

5. Aivazian, G. et al. Magnetic control of valley pseudospin in monolayer $\mathrm{WSe}_{2}$. Nat. Phys. 11, 148-152 (2015)

6. Xiao, D., Liu, G.-B., Feng, W., Xu, X. \& Yao, W. Coupled spin and valley physics in monolayers of $\mathrm{MoS}_{2}$ and other group-VI dichalcogenides. Phys. Rev. Lett. 108, 196802 (2012).

7. Hsu, W.-T. et al. Optically initialized robust valley-polarized holes in monolayer WSe $\mathrm{W}_{2}$. Nat. Commun. 6, 8963 (2015).

8. Xie, L. \& Cui, X. Manipulating spin-polarized photocurrents in $2 \mathrm{D}$ transition metal dichalcogenides. Proc. Natl Acad. Sci. USA 113, 3746-3750 (2016).

9. Cao, T. et al. Valley-selective circular dichroism of monolayer molybdenum disulphide. Nat. Commun. 3, 887 (2012).

10. Zeng, H., Dai, J., Yao, W., Xiao, D. \& Cui, X. Valley polarization in $\mathrm{MoS}_{2}$ monolayers by optical pumping. Nat. Nanotech. 7, 490-493 (2012).

11. Mak, K. F., He, K. L., Shan, J. \& Heinz, T. F. Control of valley polarization in monolayer $\mathrm{MoS}_{2}$ by optical helicity. Nat. Nanotech. 7, 494-498 (2012).

12. Yu, H., Liu, G.-B., Gong, P., Xu, X. \& Yao, W. Dirac cones and Dirac saddle points of bright excitons in monolayer transition metal dichalcogenides. Nat. Commun. 5, 3876 (2014).

13. Zhu, C. et al. Exciton valley dynamics probed by Kerr rotation in $\mathrm{WSe}_{2}$ monolayers. Phys. Rev. B 90, 161302 (2014).

14. Wang, G. et al. Valley dynamics probed through charged and neutral exciton emission in monolayer WSe $\mathrm{W}_{2}$. Phys. Rev. B 90, 075413 (2014).

15. Moody, G. et al. Intrinsic homogeneous linewidth and broadening mechanisms of excitons in monolayer transition metal dichalcogenides. Nat. Commun. 6, 8315 (2015).

16. Wang, H. et al. Radiative lifetimes of excitons and trions in monolayers of the metal dichalcogenide $\mathrm{MoS}_{2}$. Phys. Rev. 93, 045407 (2016).

17. Kang, J., Tongay, S., Zhou, J., Li, J. \& Wu, J. Band offsets and heterostructures of two-dimensional semiconductors. Appl. Phys. Lett. 102, 012111 (2013).

18. Kośmider, K. \& Fernández-Rossier, J. Electronic properties of the $\mathrm{MoS}_{2}-\mathrm{WS}_{2}$ heterojunction. Phys. Rev. B 87, 075451 (2013).

19. Hong, X. et al. Ultrafast charge transfer in atomically thin $\mathrm{MoS}_{2} / \mathrm{WS}_{2}$ heterostructures. Nat. Nanotech. 9, 682-686 (2014).

20. Ceballos, F., Bellus, M. Z., Chiu, H.-Y. \& Zhao, H. Ultrafast charge separation and indirect exciton formation in a $\mathrm{MoS}_{2}-\mathrm{MoSe}_{2}$ van der Waals heterostructure. ACS Nano 8, 12717-12724 (2014).

21. Furchi, M. M., Pospischil, A., Libisch, F., Burgdörfer, J. \& Mueller, T. Photovoltaic effect in an electrically tunable van der Waals heterojunction. Nano Lett. 14, 4785-4791 (2014).

22. Lee, C.-H. et al. Atomically thin $\mathrm{p}-\mathrm{n}$ junctions with van der Waals heterointerfaces. Nat. Nanotech. 9, 676-681 (2014).

23. Huo, N. et al. Novel and enhanced optoelectronic performances of multilayer $\mathrm{MoS}_{2}-\mathrm{WS}_{2}$ heterostructure transistors. Adv. Funct. Mater. 24, 7025-7031 (2014).

24. Rivera, P. et al. Observation of long-lived interlayer excitons in monolayer $\mathrm{MoSe}_{2}-\mathrm{WSe}_{2}$ heterostructures. Nat. Commun. 6, 6242 (2015).
25. Yu, H., Wang, Y., Tong, Q., Xu, X. \& Yao, W. Anomalous light cones and valley optical selection rules of interlayer excitons in twisted heterobilayers. Phys. Rev. Lett. 115, 187002 (2015).

26. Rivera, P. et al. Valley-polarized exciton dynamics in a 2D semiconductor heterostructure. Science 351, 688-691 (2016).

27. Maialle, M. Z., de Andrada e Silva, E. A. \& Sham, L. J. Exciton spin dynamics in quantum wells. Phys. Rev. B 47, 15776-15788 (1993).

28. Moody, G., Schaibley, J. \& Xu, X. Exciton dynamics in monolayer transition metal dichalcogenides. JOSA B 33, C39-C49 (2016).

29. Wang, G. et al. Giant enhancement of the optical second-harmonic emission of $\mathrm{WSe}_{2}$ monolayers by laser excitation at exciton resonances. Phys. Rev. Lett. 114, 097403 (2015).

30. Seyler, K. L. et al. Electrical control of second-harmonic generation in a WSe monolayer transistor. Nat. Nanotech. 10, 407-411 (2015).

31. Li, Y. et al. Probing symmetry properties of few-layer $\mathrm{MoS}_{2}$ and h-BN by optical second-harmonic generation. Nano Lett. 13, 3329-3333 (2013).

32. Kumar, N. et al. Second harmonic microscopy of monolayer $\mathrm{MoS}_{2}$. Phys. Rev. B 87, 161403 (2013).

33. Zomer, P., Guimarães, M., Brant, J., Tombros, N. \& van Wees, B. Fast pick up technique for high quality heterostructures of bilayer graphene and hexagonal boron nitride. Appl. Phys. Lett. 105, 013101 (2014).

34. Schaibley, J. R. et al. Population pulsation resonances of excitons in monolayer $\mathrm{MoSe}_{2}$ with sub-1 $\mu \mathrm{eV}$ linewidths. Phys. Rev. Lett. 114, 137402 (2015).

35. Rigosi, A. F., Hill, H. M., Li, Y., Chernikov, A. \& Heinz, T. F. Probing interlayer interactions in transition metal dichalcogenide heterostructures by optical spectroscopy: $\mathrm{MoS}_{2} / \mathrm{WS}_{2}$ and $\mathrm{MoSe}_{2} / \mathrm{WSe}_{2}$. Nano Lett. 15, 5033-5038 (2015).

36. Yu, H., Cui, X., Xu, X. \& Yao, W. Valley excitons in two-dimensional semiconductors. Natl Sci. Rev. 2, 57-70 (2015).

37. Fogler, M., Butov, L. \& Novoselov, K. High-temperature superfluidity with indirect excitons in van der Waals heterostructures. Nat. Commun. 5, 4555 (2014).

\section{Acknowledgements}

This work is mainly supported by the Department of Energy, Basic Energy Sciences, Materials Sciences and Engineering Division (DE-SC0008145 and SC0012509). H.Y. and W.Y. are supported by the Croucher Foundation (Croucher Innovation Award), the RGC of Hong Kong (HKU17305914P), and the HKU ORA. J.Y. and D.M. are supported by US DoE, BES, Materials Sciences and Engineering Division. K.W. and T.T. acknowledge support from the Elemental Strategy Initiative performed by the MEXT, Japan and a Grant-in-Aid for Scientific Research on Innovative Areas 'Science of Atomic Layers' from JSPS. P.R. and X.X. acknowledge support from the State of Washington funded Clean Energy Institute. X.X. also acknowledges a Cottrell Scholar Award, and support from the Boeing Distinguished Professorship in Physics.

\section{Author contributions}

X.X. and W.Y. conceived and supervised the project. J.S., P.R. and K.S. fabricated the devices. J.S. performed measurements, assisted by P.R. K.S. performed and analysed the polarization and phase-sensitive second-harmonic generation measurements. J.S., H.Y., X.X. and W.Y. analysed the data. J.Y. and D.G.M. provided and characterized the bulk $\mathrm{MoSe}_{2}$ and $\mathrm{WSe}_{2}$ crystals. T.T. and K.W. provided B.N. crystals. J.S., X.X., W.Y. and H.Y. wrote the paper. All authors discussed the results.

\section{Additional information}

Supplementary Information accompanies this paper at http://www.nature.com/ naturecommunications

Competing financial interests: The authors declare no competing financial interests

Reprints and permission information is available online at http://npg.nature.com/ reprintsandpermissions/

How to cite this article: Schaibley, J. R. et al. Directional interlayer spin-valley transfer in two-dimensional heterostructures. Nat. Commun. 7, 13747 doi: 10.1038/ncomms13747 (2016).

Publisher's note: Springer Nature remains neutral with regard to jurisdictional claims in published maps and institutional affiliations.

cc (i) This work is licensed under a Creative Commons Attribution 4.0 Br International License. The images or other third party material in this article are included in the article's Creative Commons license, unless indicated otherwise in the credit line; if the material is not included under the Creative Commons license, users will need to obtain permission from the license holder to reproduce the material. To view a copy of this license, visit http://creativecommons.org/licenses/by/4.0/

C The Author(s) 2016 